\section{CNN based CSI acquisition for FDD massive MIMO with noisy feedback}

\section{Qiang Sun, Yezeng Wu, Jue Wang, Chen Xu, Kai-Kit Wong}

In frequency-division-duplex (FDD) massive multiple-input multipleoutput (MIMO) systems, noisy feedback is a constant challenge for the base station (BS) to acquire accurate downlink channel state information (CSI). In this letter, we propose a convolutional neural network (CNN) based approach to overcome this problem, which we refer to it as an anti-noise CSI acquisition network (ANCAN). Results demonstrate that ANCAN can reconstruct CSI more accurately than other emerging CSI acquisition methods in the presence of noisy feedback links.

Introduction: Uplink-downlink channel reciprocity is a crucial property to estimate downlink channel state information (CSI) from the uplink for massive multiple-input multiple-output (MIMO) antenna systems where a large number of channel coefficients need to be estimated. In frequency-division-duplex (FDD) systems, nevertheless, such reciprocity no longer holds. This practically means that the downlink channel state information (CSI) has to be obtained through feedback from the users [1-3]. Conventional approaches utilise techniques such as beam domain transmission [4], long-term statistics feedback [5], compressed sensing [6], among others, to reduce the feedback overhead and increase the CSI accuracy. Deep learning (DL) has also emerged as a promising technique to design reliable CSI feedback in [7]. The so-called CsiNet in [7] is the most powerful state-of-the-art for CSI feedback which can reduce the feedback overhead while still achieving very high CSI accuracy.

The problem is that most of the techniques were not designed for noisy environments, meaning that their performance may degrade severely in the presence of noise. This motivates the work of this letter where we construct a convolutional neural network (CNN) based CSI acquisition framework to address the problem of CSI compression and robust CSI reconstruction with noisy feedback. This proposed approach is referred to as the anti-noise CSI acquisition network (ANCAN). Numerical results show that the proposed ANCAN is robust and achieves a much better CSI acquisition accuracy in the presence of noise.

System model: Consider a massive MIMO system in the downlink with $N$ transmit antennas at the base station (BS) and a single antenna user equipment (UE). We assume orthogonal frequency-division-multiplexing (OFDM) transmission with $M$ subcarriers. The channel matrix in the spatial frequency domain is expressed as

$$
\mathbf{H}=\left[\mathbf{h}_{1} \cdots \mathbf{h}_{M}\right]^{H},
$$

where $\mathbf{h}_{n} \in \mathbb{C}^{N \times 1}$ represents the channel vector on the $n$th subcarrier The CSI is estimated at the UE and obtained as $\mathbf{H}$, then fed back to the BS via a noisy feedback channel. To focus on the feedback design, we assume that the perfect channel estimation $\mathbf{H}$ can be obtained at UE.

To compress the feedback information, the channel estimation is first encoded via a well trained encoder, $g_{e}(\cdot)$,

$$
\mathbf{x}_{\mathrm{u}}=g_{e}(\mathbf{H}),
$$

where $\mathbf{x}_{\mathrm{u}} \in \mathbb{C}^{1 \times L}$ denotes the encoded codeword. After that, $\mathbf{x}_{\mathrm{u}}$ is sent to the BS through a noisy feedback link. In what follows, the BS receives the feedback codeword as

$$
\mathbf{x}_{\mathrm{b}}=\mathbf{x}_{\mathrm{u}}+\mathbf{n},
$$

where $\mathbf{n} \in \mathbb{C}^{1 \times L}$ denotes the the additive white Gaussian noise (AWGN). Then $\hat{\mathbf{H}}$ can be reconstructed from $\mathbf{x}_{\mathrm{b}}$ at the BS via a decoder, $g_{d}(\cdot)$, as

$$
\hat{\mathbf{H}}=g_{d}\left(\mathbf{x}_{\mathrm{b}}\right) \text {. }
$$

ANCAN: Note that a channel matrix can be interpreted as a $2 \mathrm{D}$ image. Since CNN has revealed promising performance in image processing, we anticipate that a CNN based ANCAN can be effective in dealing with the compression and recovery of the channel matrix. The architecture of the proposed ANCAN is illustrated in Fig. 1, which consists of an encoder and a decoder, while they are linked by a noisy feedback channel.

Encoder: The input of encoder is the real and imaginary parts of $\mathbf{H}$, first pass through a convolution layer to extract the features of $\mathbf{H}$ with kernels sized as $3 \times 3$, and then through a reshape layer to generate a

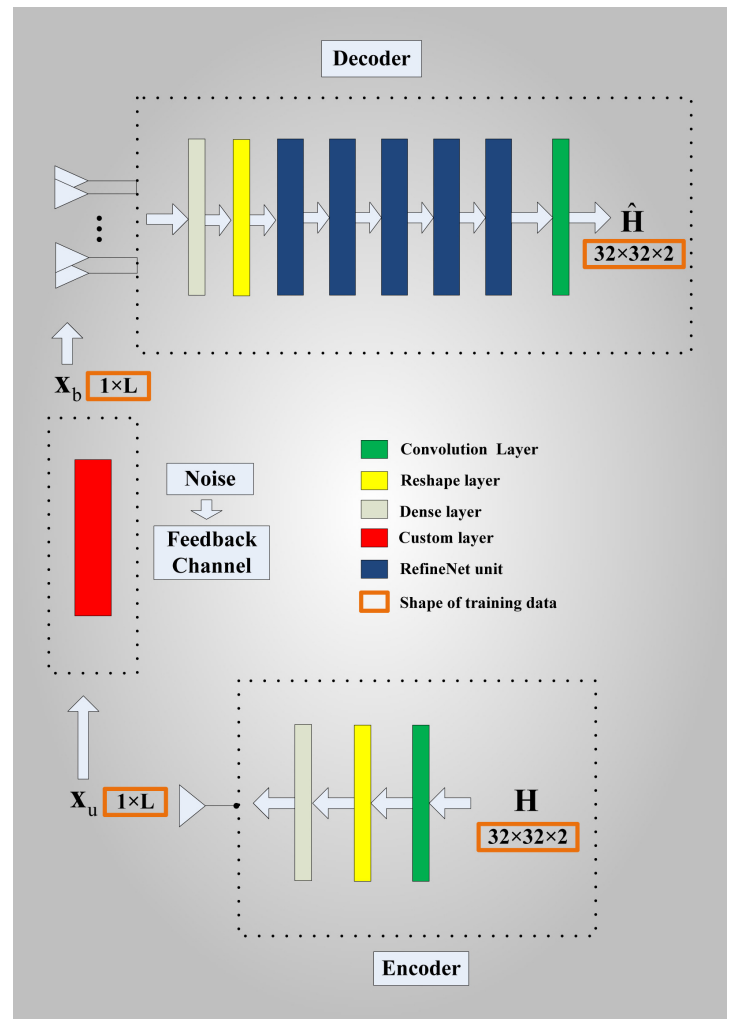

Fig. 1. The framework of ANCAN

vector. Finally a fully connected layer is used to generate the codeword $\mathbf{x}_{\mathrm{u}}$. The number of neurons in the fully connected layer represents the length of the codeword as $L$. The three layers serve as CSI compression.

Noisy feedback: A custom layer is introduced to simulate the AWGN feedback channel in the training. The custom layer allows the CNN to be trained to best fit the training data while noise in the feedback channel is considered.

Decoder: The codeword $\mathbf{x}_{\mathrm{b}}$ passes through a fully connected layer and a reshape layer to initially recover the real and imaginary parts of H. Then it can be decoded by several RefineNet units [7]. The number of RefineNet units can vary according to actual performance. Experiment results demonstrate that 5 RefineNet units provide the best performance in our considered scenario. After passing through all RefineNet units, $\hat{\mathbf{H}}$ can be finally reconstructed via the last convolution layer.

By treating $\mathbf{H}$ as an input, it passes through our proposed ANCAN as shown in Fig. 1. The parameters of the layers in the training process are denoted as $\alpha_{e}, \alpha_{n}$ and $\alpha_{d}$, respectively, and the output can be represented by $\hat{\mathbf{H}}=g_{d}\left(g_{f}\left(g_{e}\left(\mathbf{H} ; \alpha_{e}\right) ; \alpha_{n}\right) ; \alpha_{d}\right)$. The parameter updating scheme we use in the training process is the adaptive moment estimation (ADAM) algorithm in [8], and the loss function is the mean squared error (MSE).

Our results reveal that CsiNet is not designed to recover CSI from a noisy feedback link. Thus, we utilize the RefineNet units to establish ANCAN, to effectively deal with the noisy CSI feedback issue.

Experiment: The channel matrices for the training were created through the COST 2100 channel model [9], where the frequency band is setting as $5.3 \mathrm{GHz}$. The BS is equipped with $N=32$ antennas, and the number of subcarriers is $M=1024$. Moreover, the number of training samples and testing samples are considered as 100,000 and 20,000, respectively. All testing samples are excluded from the training samples. In addition, we further assume that the number of epochs is 1000, the learning rate is 0.001 , and the batch size is 200 . We compare the proposed ANCAN with the CsiNet under a noisy feedback channel.

The similarity between the input channel matrices $\mathbf{H}$ and output channel matrices $\hat{\mathbf{H}}$ can be measured by the normalized MSE (NMSE), given by

$$
\mathrm{NMSE}=\mathbb{E}\left\{\|\mathbf{H}-\hat{\mathbf{H}}\|_{2}^{2} /\|\mathbf{H}\|_{2}^{2}\right\} .
$$

Therefore, we can also have the similarity, defined as $\rho$ :

$$
\rho=\mathbb{E}\left\{\frac{1}{M} \sum_{n=1}^{M} \frac{\left|\hat{\mathbf{h}}_{n}^{H} \mathbf{h}_{n}\right|}{\left\|\hat{\mathbf{h}}_{n}\right\|_{2}\left\|\mathbf{h}_{n}\right\|_{2}}\right\},
$$




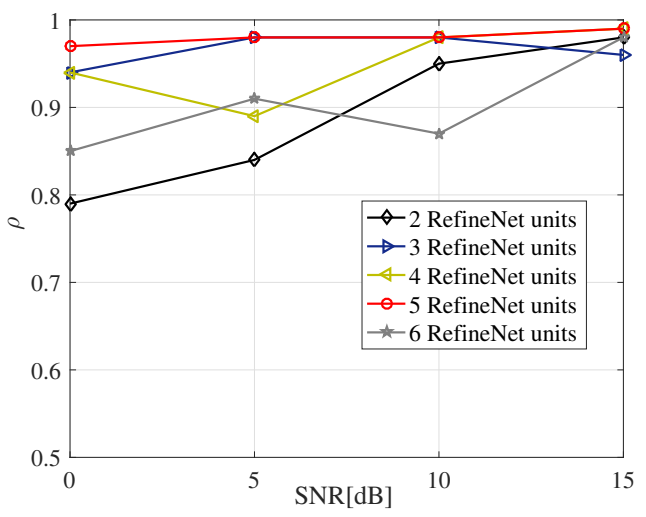

Fig. 2. $\rho$ versus SNR under different numbers of RefineNet unit

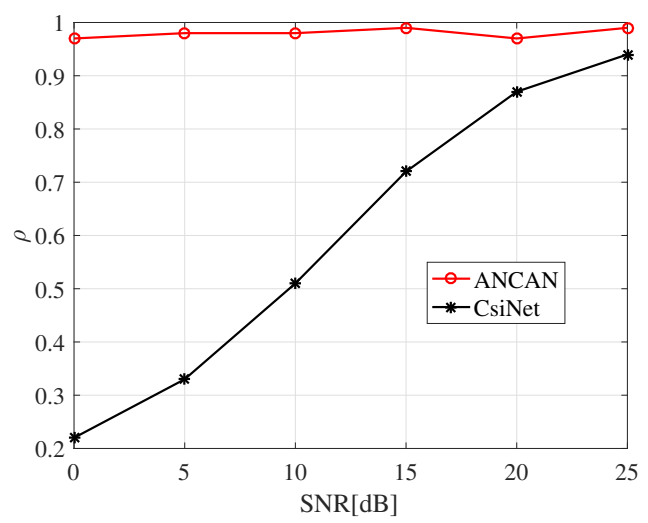

Fig. 3. Comparision of ANCAN and CsiNet with noisy feedback

where $\mathbf{h}_{n}$ and $\hat{\mathbf{h}}_{n}$ are the desired and recovered channel matrices of the $n$th subcarrier, respectively. NMSE and $\rho$ are the parameters for evaluating the quality of the noisy CSI feedback system.

Table 1: Comparison between CsiNet and ANCAN when $L=512$

\begin{tabular}{|c|c|c|c|c|}
\hline \multirow{2}{*}{ NNR[dB] Networks } & \multicolumn{2}{|c|}{ CsiNet } & \multicolumn{2}{c|}{ ANCAN } \\
\cline { 2 - 5 } & NMSE & $\rho$ & NMSE & $\rho$ \\
\hline 0 & 13.73 & 0.22 & -13.31 & 0.97 \\
\hline 5 & 9.39 & 0.33 & -14.19 & 0.98 \\
\hline 10 & 4.56 & 0.51 & -14.61 & 0.98 \\
\hline 15 & -0.27 & 0.72 & -18.51 & 0.99 \\
\hline 20 & -5.01 & 0.87 & -13.90 & 0.97 \\
\hline 25 & -9.34 & 0.94 & -18.21 & 0.99 \\
\hline
\end{tabular}

Table I compares the performance of CsiNet and ANCAN in terms of NMSE and $\rho$ when $L=512$. From $0 \mathrm{~dB}$ to $25 \mathrm{~dB}$, the NMSE and $\rho$ of ANCAN are superior to CsiNet significantly. CsiNet recovers the CSI without considering the effects of noisy feedback, even for SNR as $25 \mathrm{~dB}$, CsiNet cannot achieve the optimal performance. In contrast, ANCAN can obtain an excellent performance even if SNR is $0 \mathrm{~dB}$, while the NMSE appears to be the minimum at $15 \mathrm{~dB}$ of SNR. Clearly, ANCAN is more robust for the noisy CSI feedback.

Fig. 2 shows the results of $\rho$ for various number of RefineNet units when $L$ is set to be 512 again. We see that 5 RefineNet units provide the highest similarity. Especially at a low SNR, 5 RefineNet units are more robust to the noise effect in the feedback link, and are best for the decoder part in the ANCAN model.

The similarities of CsiNet and ANCAN are compared in Fig. 3 under the noisy feedback against the SNR. One can see that the similarities of ANCAN are much higher than CsiNet, especially in the low SNR regime. The provided results confirm that the noisy feedback has a great impact on the performance of CsiNet , which supports that our proposed ANCAN can effectively handle with the effects of the noisy feedback.

Fig. 4 demonstrates the comparison of similarities with different $L$ for the SNR as $5 \mathrm{~dB}$. ANCAN is compared with CsiNet with and without noise. $L$ is set as $32,64,128,256$ and 512, respectively. From Fig. 4, it

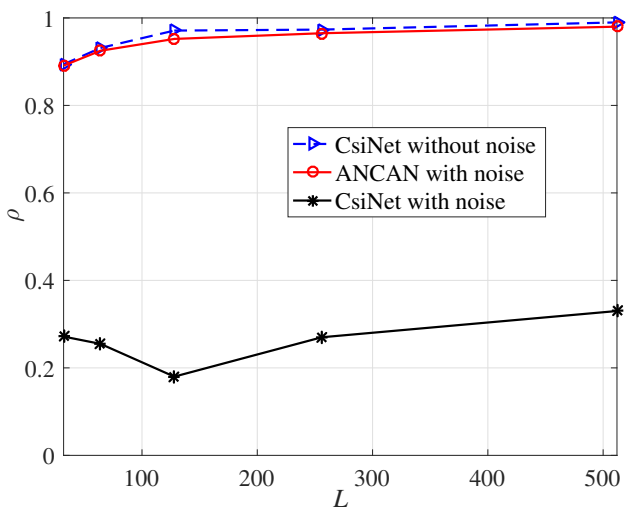

Fig. 4. $\rho$ versus different compressed codeword length $L$

is clear that regardless of the length of compressed codeword, ANCAN achieves the performance as good as CsiNet without noise (or perfect CSI feedback). Results of CsiNet illustrate that noise greatly affects its performance. The provided results indicate that ANCAN still works well under the effect of noisy feedback for different $L$. The performance of ANCAN also is improved along with an increase of $L$, approaching to that of the perfect scenario. Considering the overhead of feedback, when $L$ is set as 128 , ANCAN can achieve relatively high similarity and reduce the feedback overhead significantly.

Conclusion: In this letter, we studied the acquisition of CSI at the BS by constructing a noisy CSI feedback model in the FDD massive MIMO system. We proposed a CNN based ANCAN to reconstruct the downlink CSI at the BS with noisy feedback link. Experiment results demonstrated that the proposed ANCAN is suitable for different SNR and $L$, and recovers the CSI more accurately, which greatly improves the performance of BS for the downlink CSI acquisition.

Acknowledgment: This work was partially supported by the National Natural Science Foundation of China under Grant 61501264, 61771264,and the Nantong University-Nantong Joint Research Center for Intelligent Information Technology under Grant No. KFKT2017B01.

Qiang Sun, Jue Wang, Chen Xu (School of Electronic and Information Engineering, Nantong University, Nantong 226019, China, Nantong Research Institute for Advanced Communication Technologies (NRIACT), China)

\section{References}

1 Luo, X., Cai, P., Zhang, X., et al.: 'A scalable framework for CSI feedback in FDD massive MIMO via DL path aligning', Trans. Signal Process., $2017, \mathbf{6 5},(18)$, pp. $4702-4716$

2 Zhang, F., Sun, S., Gao, Q., et al.: 'Enhanced CSI acquisition for FDD multi-user massive MIMO systems', Access, 2018, 6, pp. 23034-23042

3 Zheng, F., Chen, Y., Pang, B., et al.: 'An efficient CSI feedback scheme for dual-polarized massive MIMO', Access, 2018, 6, pp. 23420-23430

4 Sun, C., Gao, X., Jin, S., et al.: 'Beam division multiple access transmission for massive MIMO communications', Trans. Commun., 2015, 63, (6), pp. 2170-2184

5 Wang, J., Jin, S., Gao, X., et al.: 'Statistical eigenmode-based SDMA for two-user downlink', Trans. Signal Process., 2012, 60, (10), pp. 5371-5384

6 Rao, X. and Lau, V. K. N.: 'Distributed Compressive CSIT Estimation and Feedback for FDD Multi-User Massive MIMO Systems', Trans. Signal Process., 2014, 62, (12), pp. 3261-3271

7 Wen, C., Shih, W., and Jin, S.: 'Deep learning for massive MIMO CSI feedback', Trans. Wireless Commun. Lett., 2018, 7, (5), pp. 748-751

8 Kingma, D. P., and Ba, J.: 'Adam: a method for stochastic optimization'. Proceedings of the 3rd International Conf. on Learning Representations (ICLR), 2015, pp. 1-15

9 Liu, L., Poutanen, J., Franois, Q., et al.: 'The COST 2100 MIMO channel model', Trans. Wireless Commun., 2012, 19, (6), pp. 92-99 